\title{
How Building and District Algorithms Enhance Renewable Energy Integration in Energy Markets
}

\author{
Lluc Canals Casals, Cristina Corchero, Joana Ortiz, \\ Jaume Salom, David Cardoner, Lucía Igualada \\ ECOS research group \\ Catalonia Institute for Energy Research (IREC) \\ Sant Adrià de Besòs, Spain \\ lcanals@irec.cat
}

\begin{abstract}
This study shows the results of the SABINA H2020 project, which analyzes the effect of two level optimization algorithms to increase the consumption of renewable power sources and reduce greenhouse gas emissions. First, at building level, a building algorithm maximizes the self-consumption of generated energy by its own renewable power sources. Second, at district level, a market integrated district algorithm takes into account aspects related to the electricity grid, such as the electricity generation mix and the prices of electricity and ancillary services, and aggregates the energy flexibility forecast of buildings to minimize the overall $\mathrm{CO}_{2}$ emissions while ensuring a cost reduction to prosumers.
\end{abstract}

Index Terms-- Renewable Energy, Optimization, Aggregator, Demand Response, Emissions mitigation.

\section{INTRODUCTION}

The decarbonization of the electricity system goes by the hand of a participation's increase of renewable power sources usage. Their entrance might follow the traditional way, with big generation power plants, or the way of the so called energy transition considering distributed energy resources (DER).

Following the second path, this study analyzes how buildings might contribute to this objective by taking the maximum profit of their energy consumption flexibility and to become a prosumer instead of simple consumers, that is, seeing buildings as DER. To do so, the SABINA solution takes advantage of the both intrinsic and extrinsic flexibility that buildings might count on. The intrinsic flexibility is closely related to efficiency and refers to the changes in energy consumption that energy management systems are capable to do using behind the meter strategies. In SABINA, this energy management system is called Building Algorithm (BA) which tries to maximize the self-consumption of the energy generated by rooftop solar panels. On the contrary, the extrinsic flexibility refers to the capability of buildings to change their consumptions in response to requests from third parties, following demand response (DR) criteria [1].

This research has been financially supported by the research and innovation programme Horizon 2020 of the European Union under the grant agreement nr. 731211 (SABINA). C. Corchero work is partially supported by the grant IJCI-2015-26650 (MICINN). Research by C. Corchero, Ll. Canals Casals, Jaume Salom and Joana Ortiz has been partially supported by the Generalitat de Catalunya (2017 SGR 1219). Y. Stauffer and R. Carrillo work was partially supported by the Industrial Service of Genève (SIG), Switzerland, through its SIG NER fund

978-1-7281-1257-2/19/\$31.00 @2019 IEEE

\author{
Rafael E. Carrillo \\ Yves Stauffer \\ CSEM \\ Neuchatel, Switzerland
}

As buildings on their own are unable to significantly affect the electricity grid, they need some kind of actor that coordinates their flexibility possibilities in order to gather a sufficient amount of energy [2]. This actor is known as aggregator and, in SABINA, it's named Market Integrated District Algorithm (MIDA).

This study describes the interaction of these two level algorithms and how the second (district) level modifies the expected behavior of the building to offer energy services to the electricity grid. The study presents the first results on laboratory conditions of the coordinated actions they take to improve the self-consumption of renewable power sources (from a building level) and to reduce the electricity mix $\mathrm{CO}_{2}$ emissions (from a district algorithm). Although an aggregator acts on many buildings, this study focusses the attention only in one building to fully understand the impact of both algorithms that manage a building.

As the main objective of the study is to analyze the market related decisions and activations, a general description of the BA will be presented but the attention will be focused in the MIDA that participates in balancing electricity markets.

\section{SYSTEM DESCRIPTION}

To effectively maximize the self-consumption and reduce emissions the system gathers measurement information from the buildings, from a meteorological station and forecasting information from third parties, such as weather, electricity grid prices and electricity grid mix.

The whole system is organized following a bottom-up topdown structure. That is, measurements and operational information from lower levels (appliances, battery, PV...) goes to the upper levels (BA and MIDA) who are in charge to decide what to do and send the control setpoints back to the lower levels. Following this configuration, the BA reads the measurements and weather forecast information from the building to project the optimal consumption baseline of all the elements in the building for the next 24 hours based on an MPC using a neural network. As the BA main goal is to maximize the self-consumption of the building, the 
mathematical expression that rules the algorithm is expressed in terms of minimization of the power injected to the electricity grid. The terms in this expression consider that, in the buildings, there are photovoltaic panels (PV), community batteries (that may act as generators or load according to the charge or discharge status), the electricity grid connection (being negative when injecting and that this is what is desired value to minimize), controllable power loads (such as electric vehicles and heating system) and other uncontrolled loads (such as lights and appliances used by the inhabitants of the building).

Once the optimization process is concluded, the BA sends the set-points of all the controllable elements for the next 15 minutes back to the building following the same criteria than in previous research [3]. This process is repeated every 15 minutes to adjust the possible deviations caused by stochastic events in the building, such as the occupancy, the arrival of an $\mathrm{EV}$, etc.

Notice that the BA normally runs the optimization without considering the upper level, the district algorithm, as it does not know when the MIDA will ask for an activation of flexibility and it may occur that this call does not even occur during the day.

In the upper level of the system, the MIDA has an overall sight of all the buildings of the district capable to provide DR services. When the MIDA considers that a building should activate its flexibility it sends a flexibility request to the BA 30 minutes prior to the activation with the amount of energy to increase or decrease, depending on the needs of the electricity grid. The BA then responds with the closest flexibility value it can achieve for that hour and, if the MIDA considers it as acceptable, it confirms the activation of the flexibility with the amount of energy indicated by the BA in the response message.

The aggregator or district algorithm follows a similar configuration to the one presented by Iria et al. [4], where it relies on the Home Energy Management Systems (HEMS) to act on the controllable elements in each building without having detailed information of what really happens in it. With this configuration, the MIDA has less direct control of what is happening but it also needs less computational efforts than with other configurations that monitor the exact status of all the elements in all the buildings of its portfolio and it controls each one of them to provide the required energy [5].

\section{A. Market integrated District Algorithm (MIDA)}

Theoretically, it is stated that in a stable situation, the higher the energy demand the higher the use of carbon intensive power sources, thus, an aggregator of demand could be useful to reduce emissions when imbalances occur [6]. Assuming that the transition towards a $100 \%$ renewable energy generation should focus on the cleanliness of the electricity generation rather than only on economics, the MIDA's goal is to minimize the greenhouse gas emissions of districts by taking advantage of the flexibility of prosumers within the district. To do so, instead on focusing in minimization of the electricity costs as most of aggregators do [7]-[8], the MIDA uses the grid electricity mix as driver, following the idea of Diekerhof et al. [9] but ensuring that no additional costs will be charged to the prosumers.

Therefore, this paper proposes a centralized aggregator solution that focus on the needs of the DSO or TSO.

MIDA is built in a modular way, having an optimization module named OPTIMIDA that runs during the day ahead, an intraday module (FLEXMAN) in charge of the activation of flexibility and a module that evaluates the results (KPtaIn) as shown schematically in Figure 1.

OPTIMIDA takes into account aspects related to the electricity grid, such as the electricity generation mix and forecasts of the prices of electricity and payments for ancillary services. This information is taken from third parties (generally the TSO). Then, it aggregates the energy flexibility forecast of prosumers given by the BA. This flexibility forecast is estimated by the building algorithm and indicates the baseline consumption and the maximum energy flexibility for the next day and the rebound effect expected due to the activations in relation to the consumption baseline (flexibility map shown in Figure 3). Then, this module optimizes a market offer by selecting which buildings are to be called to offer flexibility services each hour to minimize the overall $\mathrm{CO}_{2}$ emissions at a district level while ensuring no additional costs to prosumers.

The optimization problem that faces OPTIMIDA is described in equation (1).

THIRD PARTIES

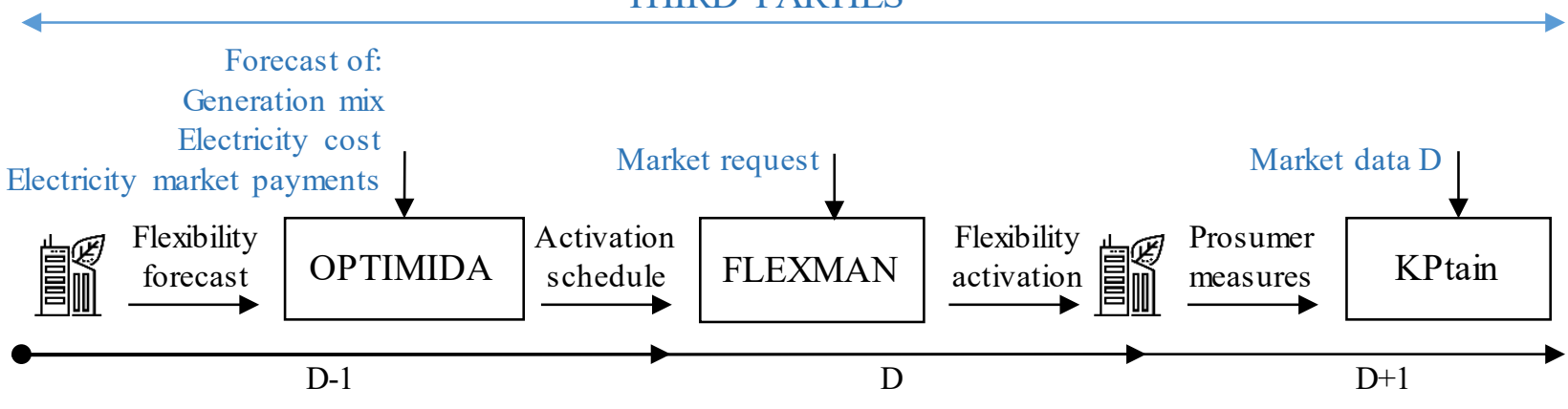

Figure 1. Operational schema of the MIDA and its modules. 


$$
\begin{aligned}
& f(D A)=\operatorname{Min} \sum_{h=0}^{24} \text { Emissions Flex } x_{h}= \\
& =\operatorname{Min} \sum_{h=0}^{24} \sum_{i=1}^{n}\left(\left(\beta_{\Delta i h} \cdot \text { Flex }_{\Delta i h} \cdot \text { Mix }_{h}\right)-\sum_{r}\left(\text { Base }_{\Delta i h r}-\text { Reb }_{\Delta i h r}\right) M i x_{h r}\right) \\
& -\left(\left(\beta_{\nabla i h} \cdot \text { Flex }_{\nabla i h} \cdot \text { Mix }_{h}\right)-\sum_{r}\left(\text { Base }_{\nabla i h r}-\text { Reb }_{\nabla i h r}\right) M i x_{h r}\right)
\end{aligned}
$$

Where $\beta_{\Delta \text { ih }}$ represents the relation between the effective flexibility (Upwards) against the maximum flexibility given by the BA during the day ahead in the flexibility map $\left(\right.$ Flex $\left._{\Delta i h}\right) . \beta_{\Delta \text { ih }}$ is equal to 1 when the DA asks for the total amount of flexibility offered by the BA and it is 0 when there is no request of flexibility. Similarly, $\beta_{\nabla \text { ih }}$, represents the effort when flexibility goes downwards. Mix ${ }_{h}$ refers to the electricity mix corresponding to a determined hour of the day and Mix to the mix at a determined hour during the rebound effect duration, while Base $_{\Delta \text { ihr }}$ and $\mathrm{Reb}_{\Delta \mathrm{ihr}}$ represent the baseline and the rebound energy consumption of a building at a determined hour during the rebound effect (Figure 3). To work well, there are several restrictions that MIDA should comply:

- the MIDA is limited to one activation per day per building (in order to reduce the interferences with the normal use of the MPC).

- During the rebound effect, no activations are allowed.

- Buildings should NOT lose money with the activation. The computation considers the incomes and costs from flexibility capacity and energy.

- Requested capacity is never higher than what the flexibility map indicated.

- The duration of an activation is defined as $1 \mathrm{~h}$.

When the MIDA receives a market request (that is, a necessity to balance the grid), the flexibility manager module (FLEXMAN) is in charge of allocating the flexibility requested in its portfolio of active buildings in real time. The module allocates flexibility based on the previous results of OPTIMIDA and considers reliability indexes calculated for each prosumer.

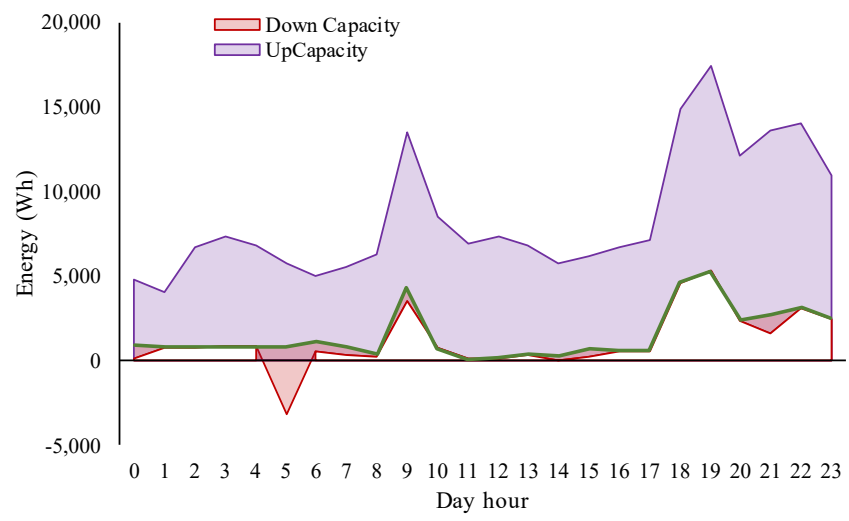

Figure 3. Example of a flexibility map with the baseline (green), upwards capacity (purple) and downwards capacity (red).

Finally, the KPtaIn module is in charge to evaluate the response of prosumers to activations and the overall impact on $\mathrm{CO}_{2}$ emissions reduction. Then, this module is in charge to update the reliability indexes of prosumers enhancing the participation of highly available prosumers with accurate consumption forecasts by an increase of their reliability index, whereas prosumers that provide inaccurate forecasts or are not available when required will decrease their index.

\section{B. Laboratories and tests}

To launch the first operational SABINA tests, two laboratories in IREC (SMARTLAB and SEILAB in Barcelona and Tarragona respectively) are used to emulate thermal and electrical elements of the simulated buildings that run on EnergyPlus models wrapped into a Functional Mock-up Unit to co-simulate on Labview software [10] to represent the thermal behavior of the building. The buildings have four dwellings, one per stage, and the elements capable of offering energy flexibility are:

- A community $10.8 \mathrm{~kW}_{\mathrm{p}}$ Photovoltaic system.

- Two $24 \mathrm{kWh}$ electric vehicles (simulated).

- A $10 \mathrm{kWh}$ community Li-ion battery (with an operative power of $4 \mathrm{~kW}$ ).

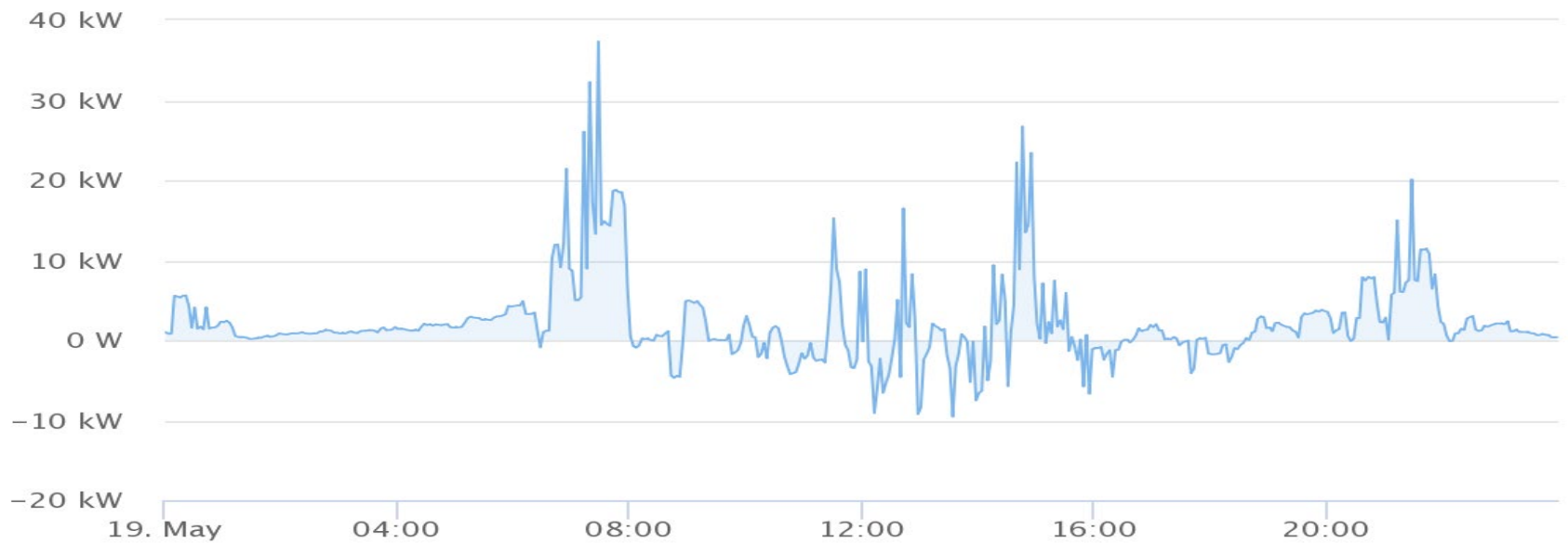

Figure 2. Screenshot of the consumption of SMARTLAB and its activation peaks taken from the project's broker website. 
- Water-to-water heat pump (Some buildings have a centralized system (real) for all the community and others an individual simulated heating system per dwelling).

\section{RESULTS AND DISCUSSION}

The first functional tests were launched on May $16^{\text {th }}$ for five days. Figure 2 shows the electricity consumption of the four floor building simulated in SMARTLAB for the day 19. Notice how the activation of flexibility by MIDA is clearly visible in the consumption curve that presents a relevant upward peak at 7A.M. in the morning.

It should be clarified that, during the 4 days test, most flexibility activations were upwards because the flexibility offered by the building in the flexibility map (Figure 3) presents higher opportunities to go in this direction rather than downwards.

The system uploads, every quarter of an hour, the updated or adjusted baseline consumption of the building. This updated baseline considers the latest real life events caused by the use of the devices in the building by the people living in them, such as the oven or the use of hot water for morning showers among others, or the activation of the heating system caused by deviations between the predictions and the real temperature and weather conditions of the day. This adjusted baseline curve is represented in purple in Figure 4 starting at the previous hour of the activation.

Entering deeper into the activation details, Figure 4 presents the divergence in the consumption of the building that the activation of flexibility (red area) represent against the BA projected consumption (purple area) for one of the days in of the test.

Notice that effectively, there is a huge difference in the consumption at $7 \mathrm{~h}$ in the morning but then, during the following hours, the real consumption of the building is lower than what it was expected to occur in the previous cases. This is the so-called rebound effect, meaning that the building has already used the energy that was expected to use later.

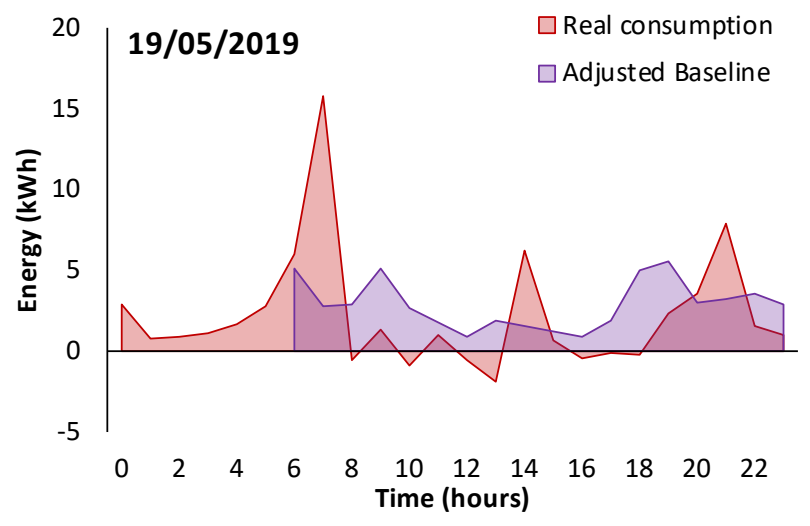

Figure 4. Real (red) and adjusted baseline (purple) consumptiom of the whole building.
In this case, most of the amount of energy of the flexibility activation came from the heat pumps dedicated to space heating, reducing the needs for building heating afterwards.

With regards to the emissions of the building, Figure 5 shows that, effectively, just at the moment of flexibility activation, the emissions of $\mathrm{CO}_{2}$ related to the consumption of electricity from the grid of the building is much higher than what was expected with the normal use. Then, hour after hour, this difference gets lower until they met again at $13 \mathrm{~h}$. Finally, at 18 hours the "expected" consumption of the building presents a peak that makes the corresponding emissions rise finishing the day with higher emissions than what occurs thanks to the application of flexibility.

As seen, the amount of emissions reduced thanks to the activation of flexibility corresponds to almost $18 \%$ for this day.

From an economic perspective, the activation of flexibility has its impact. For this particular case, the cost of energy at the end of the day was of $5.80 €$ for the whole building. In comparison to the expected result from the building algorithm's baseline that would have resulted in $6.01 €$, there are 21 cents discount that represents a cost reduction of $3 \%$.

Note that the goal of SABINA is to enhance the entrance of renewable energy. This is why the BA tries to maximize the self-consumption (avoiding injection of energy to the grid) and, in parallel, MIDA tries to minimize the emissions taking profit of the electricity grid. The mixture of both shows how MIDA forces the BA to inject small amounts of energy to the grid knowing that the overall result might be better.

Note, additionally, that although there are economic revenues from the activation of flexibility, they are quite lower when compared to the environmental effects. This is due to the fact that the economic aspects are conceived as a restriction (not to lose money) and not as a goal to achieve.

Finally, the presented results correspond to the particularities of one day. Results strongly depend on the inhabitants of the building and the final uses they give to the equipment within as they can deviate the consumption of the building from the baseline.

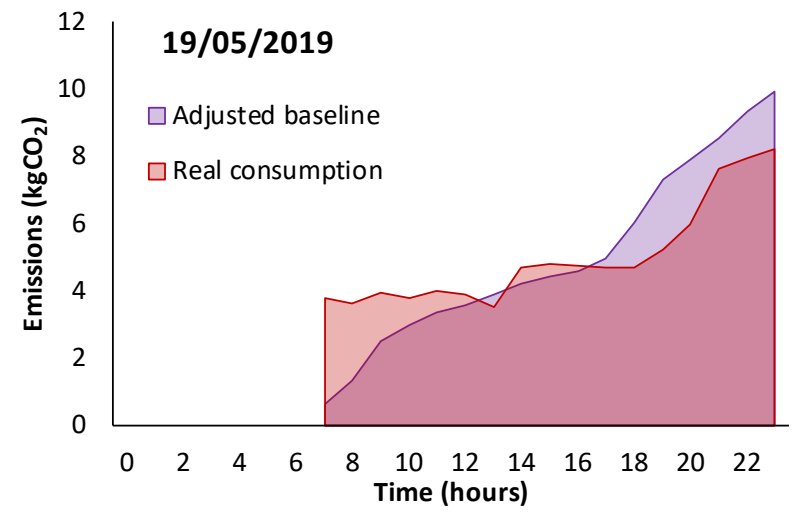

Figure 5. Accumulated $\mathrm{CO}_{2}$ emissions caused by the building energy exchange with the electricity grid. 


\section{CONCLUSIONS}

This first results of the SABINA solution shows that, effectively, balancing energy markets have the opportunity to enhance the entrance of renewable power sources into the electricity grid. Its effectiveness is clearly tied to the goals of the aggregator, if the aggregator's goal is to maximize economic profit, the reduction RES increase could be harder to achieve.

Results show that, effectively, there is a margin to reduce the emissions of a building if it interacts with the electricity grid without ending in a higher electricity costs. Therefore, environmental drivers for optimal use of energy in buildings are to be considered if an effective energy transition is to be done instead of focusing most of the attention into the economics.

Moreover, the two level algorithm implemented in SABINA shows how the results of the optimization of the upper level might significantly affect the results of the lower level algorithm. In the example presented in this study, the goal of the building algorithm, which minimizes the injection of energy to the grid, was perfectly achieved without the participation of the aggregator (no injection at all was predicted). However, after the aggregator activates the flexibility of the building to reduce the overall $\mathrm{CO}_{2}$ emissions, there are several hours of the day when the building injects electricity to the grid caused by the reduction of inherent flexibility resources previously consumed by the district algorithm.

\section{REFERENCES}

[1]

[2]
M. Behrangrad, "A review of demand side management business models in the electricity market," Renew. Sustain. Energy Rev., vol. 47, pp. 270-283, 2015.

L. Canals Casals, M. Barbero, and C. Corchero, "Reused second life batteries for aggregated demand response services," J. Clean. Prod., vol. 212, pp. 99-108, 2019.

T. Pean, R. Costa-Castello, E. Fuentes, and J. Salom, "Experimental Testing of Variable Speed Heat Pump Control Strategies for Enhancing Energy Flexibility in Buildings," IEEE Access, vol. 7, pp. 37071-37087, 2019.

J. Iria, F. Soares, and M. Matos, "Optimal supply and demand bidding strategy for an aggregator of small prosumers," Appl. Energy, no. June, pp. 1-12, 2017.

P. Olivella-Rosell et al., "Optimization problem for meeting distribution system operator requests in local flexibility markets with distributed energy resources," Appl. Energy, vol. 210, no. April, pp. 881-895, 2017.

E. Mckenna and S. J. Darby, "How much could domestic demand response technologies reduce $\mathrm{CO} 2$ emissions?," in ECEEE on Consumption, efficiency and limits, 2017, pp. 337-347.

C. Shao, Y. Ding, J. Wang, and Y. Song, "Modeling and Integration of Flexible Demand in Heat and Electricity Integrated Energy System,” IEEE Trans. Sustain. Energy, vol. 3029, 2017.

R. Henriquez Auba, G. Wenzel, and D. Olivares, "Participation of Demand Response Aggregators in Electricity Markets: Optimal Portfolio Management," IEEE Trans. Smart Grid, 2017.

M. Diekerhof, F. Peterssen, and A. Monti, "Hierarchical Distributed Robust Optimization for Demand Response Services," IEEE Trans. Smart Grid, vol. 3053, no. c, pp. 1-1, 2017.

P. Taddeo, I. Bellanco, J. Ortiz, E. Fuentes, and J. Salom, "Realtime experimental connection of an EnergyPlus building model with a real water-te-water heat pump through Functional Mock-up Interface," in Building Simulation, 2019. 